\title{
FORMULACIÓN Y RESOLUCIÓN GRÁFICA DE PROGRAMAS MATEMÁTICOS
}

Responsable: Lic. Luis Méndez Ávalos Miembro: Msc. Jhony Chávez Delgado

Miembro: Mgr. Eduardo Rodríguez Delgado

\section{RESUMEN}

El presente articulo establece condiciones para la existencia de soluciones óptimas de programas matemáticos mediante condiciones de globalidad de los teoremas de Weierstrass y fundamental de la programación convexa, debido a la limitación que presenta el método de resolución gráfica cuando el número de variables de decisión es mayor que dos, para lo cual disponemos de criterios generales que garanticen la existencia de solución óptima de un programa matemático.

El análisis gráfico nos ayuda a comprender resultados sobre cómo varian las soluciones óptimas de un programa cuando se modifica la función objetivo o el conjunto de soluciones factibles. Asi mismo, las diferentes definiciones y técnicas permiten aplicar la optimización matemática a la economía. Finalmente, el presente artículo es un aporte al estudio de la optimización sin restricciones.

\begin{abstract}
The present article of investigation establishes conditions for the existence of optimal solutions of mathematical programs by means of conditions of globalidad of the theorems of fundamental Weierstrass and of the convex programming, due to the limitation that presents/displays the method of graphical resolution when the number of decision variables is greater than two, which we have general criteria that they guarantee the existence of optimal solution of a mathematical program.
\end{abstract}

\section{INTRODUCCIÓN}

En la teoria de la optimización muchos problemas que se plantean tienen una estructura lógica común, ya que la idea es encontrar la mejor solución posible en determinadas circunstancias. Dicha solución permitirá decidir las medidas a tomar para alcanzar los objetivos que se persiguen. Este tipo de problemas puede aparecer en geometria, estadistica, economía, etc.

Ante un problema de optimización, parece lógico plantearse, en primer término, sobre qué variable podemos decidir. Una vez identicadas las variables de decisión nos preguntaremos cuál es el criterio que nos permitirá elegir la mejor decisión, de acuerdo con el objetivo que se pretende alcanzar. Por último, es conveniente conocer cuáles son las distintas opciones disponibles, es decir, los valores posibles de las variables de decisión según la naturaleza del problema al que nos enfrentamos. Tras especificar el problema de optimización estamos en condiciones de abordar su resolución.

Una vez expresado en términos matemáticos el problema que pretendemos resolver, nos preguntamos si tiene solución y en caso afirmativo, cuál es su localización. Cuando tenemos un programa matemático con dos variables de decisión, su resolución gráfica puede darnos la respuesta a las dos cuestiones anteriores, permitiéndonos, además, discernir si la solución encontrada es local o global.

Dada la limitación que presenta el método de resolución gráfica cuando el número de variables de decisión es mayor que dos, conviene disponer de criterios generales que garanticen la existencia de solución óptima de un programa matemático. Los teoremas de Weierstrass y fundamental de la Programación Convexa proporcionan respectivamente condiciones suficientes de existencia de óptimos globales y de globalidad de las soluciones de un programa matemático.

\section{FORMULACIÓN DE PROGRAMAS MATEMÁTICOS}

\subsection{Programa Matemático:}

Los programas matemáticos que vamos a formular y resolver en este artículo de Investigación admiten la siguiente formulación general:

$$
\left.\begin{array}{c}
\text { Opt } f\left(x_{1}, \ldots, x_{n}\right) \\
\text { s.a. } g_{1}\left(x_{1}, \ldots, x_{n}\right)=0 \\
\vdots \\
g_{m}\left(x_{1}, \ldots, x_{n}\right)=0 \\
h_{1}\left(x_{1}, \ldots, x_{n}\right) \leq 0 \\
\vdots \\
h_{k}\left(x_{1}, \ldots, x_{n}\right) \leq 0 \\
x=\left(x_{1}, \ldots, x_{n}\right) \in D \subset R^{n}
\end{array}\right\}
$$

$\operatorname{con} f, h_{i}, g: \mathbb{R}^{n} \rightarrow R \quad i=1, \ldots, m, j=1, \ldots, k(m<n)$ 


\subsection{Elementos de un Programa Matemático:}

Los elementos de un programa matemático son los siguientes:
a) $\left(x_{1}, \ldots, x_{r}\right)$ :Variables de decisión
b) $f(x) \quad$ :Función objetivo del problema
c) opt :Optimizar la función $\mathrm{f}$ consiste en encontrar su máximo y su mínimo.
d) $g(\bar{x})=0 \quad$ :Restricciones de igualdad que han de cumplir las posibles soluciones.
e) $h_{1}(\bar{x}) \leq 0 \quad$ :Restricciones de desigualdad que
f) $\bar{x} \in D \quad$ :Restricciones conjuntivas.

\subsection{Ejemplos de un Programa Matemático:}

Formular los programas matemáticos que permiten resolver los siguientes programas:

a) Calcular las dimensiones del rectángulo de perímetro 2 metros que tiene área máxima.

b) Un entusiasta de la salud desea consumir un mínimo de 36 unidades de vitamina $A$ al dia, 28 unidades de vitamina $C$ y 32 de vitamina D. Un determinado complejo vitamínico de marca 1 cuesta 300 soles y proporciona 2 unidades de vitamina $A, 2$ de vitamina $C$ y 8 de vitamina D. El de marca 2 cuesta 400 soles y proporciona 3 unidades de vitamina A, 2 de vitamina $C$ y 2 de $D$. Calcular la combinación de complejos vitamínicos de costo más bajo y que garantiza las necesidades diarias.

\section{Solución:}

a) Un rectángulo queda caracterizado por las dimensiones de su base y altura.

Definamos como variables de decisión:

$$
\begin{aligned}
& x=\text { base del rectángulo } \\
& y=\text { altura del rectángulo }
\end{aligned}
$$

El área del rectángulo será $A(x, y)=x . y$

El perímetro del rectángulo es

$$
P(x, y)=2 x+2 y
$$

Por lo tanto, el programa es el siguiente:

$$
\begin{aligned}
\text { Max. } \quad A(x, y) & =x . y \\
\text { s.a. } \quad 2 x+2 y & =2 \\
& \\
& \geq 0 \\
y & \geq 0
\end{aligned}
$$

b) Las variables de decisión son:

$x=$ cantidad diaria a consumir de la marca 1.

$y=$ cantidad diaria a consumir de la marca 2 .

El objetivo es minimizar la función de costo: $C(x, y)=300 x+400 y$
Restricciones que se tienen que cumplir:

- Cubrir las necesidades diarias de vitamina $A: 2 x+3 y \geq 36$

- Cubrir las necesidades diarias de vitamina $C: 2 x+2 y \geq 28$

- Cubrir las necesidades diarias de vitamina D: $8 x+2 y \geq 32$

- No negatividad de las cantidades a consumir: $x \geq 0, y \geq 0$

Por lo tanto, el programa es:

$$
\left.\begin{array}{cc}
\min & C(x, y)=300 x+400 y \\
\text { s.a } & 2 x+3 y \geq 36 \\
& 2 x+2 y \geq 28 \\
& 8 x+2 y \geq 32 \\
& x \geq 0, y \geq 0
\end{array}\right\}
$$

\section{RESOLUCIÓN GRÁFICA DE PROGRAMAS MATEMÁTICOS}

\subsection{Definiciones:}

Definición 3.1.1 Sealafunción $f: U \rightarrow R, U \in \mathbb{R}^{n}$ para cada $\quad k \in R$, se define el conjunto de nivel de kde f como:

$$
C_{k}=\{\bar{x} \in U / f(x)=k\} \subset \mathbb{R}^{n}
$$

Definición 3.1.2 Sea f $U \rightarrow \mathbb{R}, U \subset \mathbb{R}^{n}$ una función diferenciable en $U$, entonces si $\bar{x}_{0} \in U$.

el vector gradiente $\nabla f\left(\bar{x}_{0}\right)$

es perpendicular al conjunto:

$$
C_{f\left(x_{0}\right)}=\left\{x \in U / f(x)=f\left(x_{0}\right)\right\} \text {, en el punto } \bar{x}_{0}
$$

Definición 3.1.3 El conjunto factible A del problema (P) es el conjunto de todos los puntos $\bar{x} \in \mathbb{R}^{n} q u$ e verifican todas las restricciones del programa, es decir,

$$
A=\left\{\bar{x} \in \mathbf{R} / h_{1}\left(x_{1}, \ldots, x_{1}\right)=0, i=1, \ldots, m, g\left(x_{1}, \ldots, x_{0}\right) \leq 0, j=1, \ldots, \bar{x} \in D\right\}
$$

Definición 3.1.4 Dado el programa matemático

$$
\text { _ } \left.\bar{x}=\left(x_{1}, \ldots, x_{n}\right) \in A \subset R^{1}\right\}
$$

(I) Se dice que $X_{0} \in A$ es mínimo local del programa, si se verifica que :

$$
f(\bar{x}) \leq f\left(\bar{x}_{0}\right), \forall \bar{x} \in A
$$

(ii) Se dice que $X_{0} \in A$ es mínimo local del programa, si se verifica que :

$$
f(\bar{x}) \geq f\left(\bar{x}_{0}\right), \forall \bar{x} \in A
$$

(iii) Se dice que $X_{0} \in A$ es máximo local estricto del programa, si se verifica que

$$
f(\bar{x})<f\left(\bar{x}_{0}\right), \forall \bar{x} \in A \text { y } \bar{x} \neq \bar{x}_{0}
$$

(iv) Se dice que $\bar{X}_{0} \in A$ es mínimo local estricto del programa, si se verifica que

$$
f(\bar{x})>f\left(\bar{x}_{0}\right), \forall \bar{x} \in A \text { y } \bar{x} \neq \bar{x}_{0}
$$


Definición 3.1.5 Dado el programa

$$
\left.\begin{array}{c}
\text { Opt } f\left(x_{1}, \ldots, x_{n}\right) \\
\bar{x}=\left(x_{1}, \ldots, x_{n}\right) \in A \subset \mathbb{R}^{n}
\end{array}\right\}
$$

(I) Se dice que $x_{0} \in A$ es máximo local de programa, si existe $r>0$ tal que

$$
f(\bar{x}) \leq f\left(\bar{x}_{0}\right), \forall \bar{x}_{0} \in B\left(\bar{x}_{0} ; r\right) \cap A
$$

(li) Se dice que $x_{0} \in A$ es mínimo local del programa, si existe $r>0$ tal que

$$
f(\bar{x}) \geq f\left(\bar{x}_{0}\right), \forall \bar{x}_{0} \in B\left(\bar{x}_{0} ; r\right) \cap A
$$

(iii) Se dice que $\bar{X}_{0} \in A$ es máximo local estricto del programa, si existe $r>0$ tal que

$$
\mathrm{f}(\overline{\mathrm{x}})<\mathrm{f}\left(\overline{\mathrm{x}}_{0}\right), \forall \overline{\mathrm{x}}_{0} \in \mathrm{B}\left(\overline{\mathrm{x}}_{0} ; \mathrm{r}\right) \cap \mathrm{A}
$$

(iv) Se dice que $x_{0} \in A$ es mínimo local estricto del programa, si existe $r>0$ tal que

$$
f(\bar{x})>f\left(\bar{x}_{0}\right), \forall \bar{x}_{0} \in B\left(\bar{x}_{0} ; r\right) \cap A
$$

\subsection{Ejemplo:}

Resolver gráficamente el programa matemático:

$$
\begin{aligned}
& \text { Opt. }(x-2)^{2}+(y-2)^{2} \\
& \begin{array}{c}
\text { S.a. } 2 x+3 y \geq 6 \\
3 x+2 y \leq 12 \\
x \geq 0 \\
y \geq 0
\end{array}
\end{aligned}
$$

\section{Solución:}

Las curvas de nivel de la función objetivo $f(x, y)=(x-2)^{2}+(y-2)^{2}$ son circunferencias de centro en el punto $(2,2)$ $y$ radio $r=\sqrt{k}$, siendo $k>0$ el nivel de la curva.

El Conjunto Factible:

$A=\left\{(x, y) \in \mathbb{R}^{2} / 2 x+3 y \geq 6,3 x+2 y \leq 12, x \geq 0, y \geq 0\right\}$

es un cuadrilátero de vértices $(3,0),(4,0)$, $(0,6),(0,2)$ como puede verse en la figura

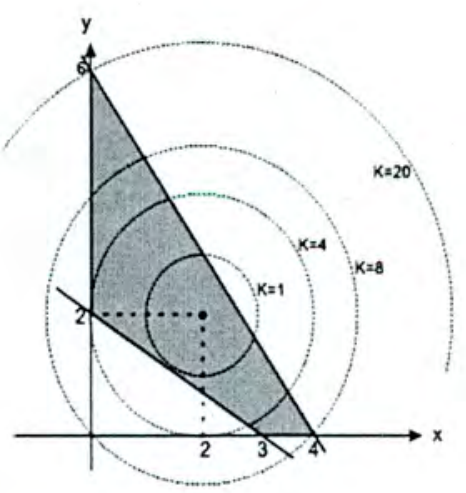

Al superponer las curvas de nivel sobre el conjunto factible, se obtiene que $x_{0}=(2,2)$ es el mínimo global $\mathrm{y}_{\mathrm{x}}=(0,6)$ el máximo global. Los valores de la función objetivo son $f(2,2)=0 y$ $f(0,6)=20$.

\section{CONDICIONES DE GLOBALIDAD}

\subsection{Teorema de Weierstrass y fundamental de la programación convexa}

Teorema 4.1 (teorema de Weierstrass)Sea $f: \mathbb{R}^{n} \rightarrow \mathbb{R}$ Una función continua en $A \subset \mathbb{R}^{n}$ $y$ sea $A$ un conjunto cerrado $y$ acotado.

Entonces existen $\bar{x}_{1}, \bar{x}_{2} \in A$ tal que:

$$
\begin{aligned}
& f\left(\bar{x}_{1}\right) \leq f(\bar{x}), \quad \forall \bar{x} \in A \\
& f\left(\bar{x}_{2}\right) \geq f(\bar{x}), \quad \forall \bar{x} \in A
\end{aligned}
$$

Es decir, $\bar{x}_{1}$ es mínimo global de fen $A$ y $\bar{x}_{2}$ es máximo global de fen $\mathrm{A}$.

Teorema 4.2 (teorema fundamental de la programación convexa): Dado el programa convexo.

$$
\left.\begin{array}{c}
\min f\left(x_{1}, \ldots, x_{n}\right) \\
\text { s.a. }\left(x_{1}, \ldots, x_{n}\right) \in A \subset \mathbb{R}^{n}
\end{array}\right\}
$$

Se dice que:

i) $S i \bar{x}_{1} \in A$ es un mínimo local, entonces $x_{1}$ es un mínimo global.

ii) El conjunto de todos los mínimos del programa es un conjunto convexo.

\subsection{Ejemplos:}

4.2.1. Dado el siguiente programa:

$$
\begin{gathered}
\text { Opt. } 3 x+y \\
\text { s.a. } x^{2}+y^{2} \leq 5 \\
x-y \leq 1 \\
x \geq 0
\end{gathered}
$$

a) Estudiar analíticamente si verifican el Teorema de Weierstrass.

b) Resolver gráficamente.

\section{Solución:}

a) Veamos si se cumplen las tres hipótesis del teorema.

1. $f(x, y)=3 x+y$ es continua por ser una función polinómica.

2. El conjunto factible es cerrado. En efecto, por ser continuas las funciones:

$g_{1}(x, y)=x^{2}+y^{2}, g_{2}(x, y)=x-y$, $g_{3}(x, y)=x$ 
Se verifica que los conjuntos son cerrados.

$$
\begin{aligned}
& g_{1}^{-1}\{(-\infty, 5]\}=\left\{(x, y) \in R^{2} / x^{2}+y^{2} \leq 5\right\} \\
& g_{2}^{-1}\{(-\infty, 1]\}=\left\{(x, y) \in R^{2} / x-y \leq 1\right\} \\
& g_{3}^{-1}\{[0, \infty)\}=\left\{(x, y) \in R^{2} / x \geq 0\right\}
\end{aligned}
$$

3. El conjunto factible está acotado, ya que dado $M=\sqrt{5}$ se verifica que

$$
\bar{x} \in A,\|\bar{x}\|=\sqrt{x^{2}+y^{2}} \leq \sqrt{5}
$$

Como se cumplen las tres condiciones de teorema de Weierstrass, el programa dado tiene máximo global y mínimo global.

b) En este caso, podemos ver gráficamente que el máximo está en el punto del primer cuadrante del corte de la circunferencia y la recta, es decir,

de donde: $\quad\left\{\begin{array}{c}x^{2}+y^{2} \leq 5 \\ x-y=1\end{array}\right.$

$$
y^{2}+y-2=0
$$

Como el punto $(-2,-1)$ no es factible, el máximo esta en el punto $(2,1)$ siendo $f(2,1)=7$. El mínimo en $(0,-1)$ y valor mínimo es $f(0,-1)=-1$.

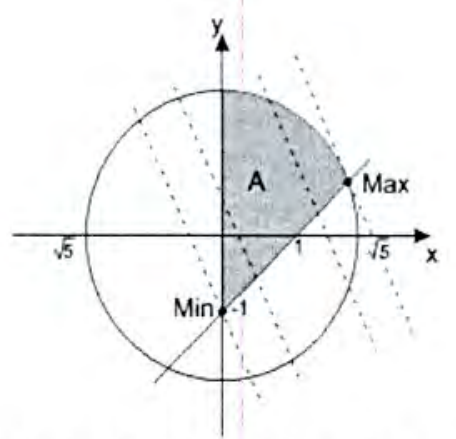

4.2.2. Dado el siguiente programa:

$$
\left.\begin{array}{ll}
\text { opt } x-y^{2} \\
\text { s.a. } & (x-1)(y-2) \geq 0 \\
2 \leq x \leq 4
\end{array}\right\}
$$

a) Resolver gráficamente

b) Teniendo en cuenta los resultados obtenidos en el apartado a), discutir cuáles pueden ser las causas por las que existe o no solución óptima.

\section{Solución:}

a)

$$
\left.\begin{array}{l}
\text { opt } x-y^{2} \\
\text { s.a. } \\
(x-1)(y-2) \geq 0 \\
2 \leq x \leq 4
\end{array}\right\}
$$

El conjunto de soluciones factibles del programa es

$$
\begin{aligned}
& B=\left\{(x, y) \in R^{2} l(x-1)(y-2) \geq 0,2 \leq x \leq 4\right\} \\
& \text { y su representación junto con la } \\
& \text { correspondiente a las curvas de nivel de la } \\
& \text { función objetivo, que son la familia de } \\
& \text { parábolas de ecuación } x=y+2 k \text {, que se } \\
& \text { indica en la figura siguiente }
\end{aligned}
$$

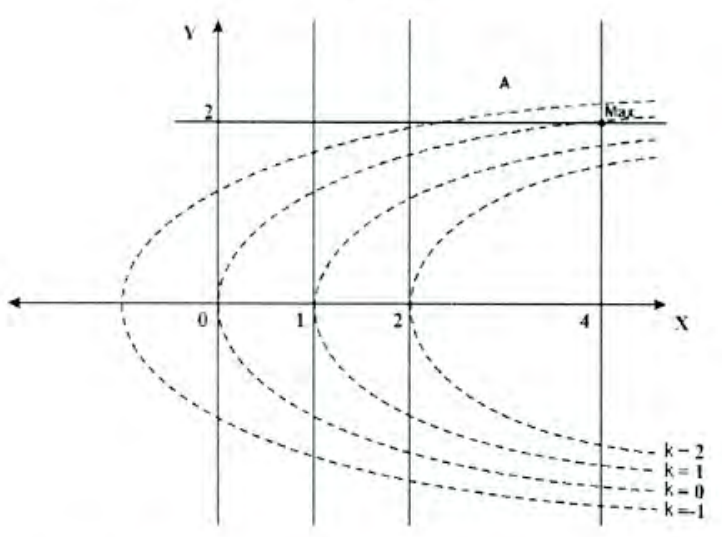

Al resolver gráficamente este programa, obtenemos que el máximo se alcanza en el punto $(4,2)$, siendo el valor máximo de la función objetivo igual a 0 . Sin embargo, no existe una solución factible en que la función objetivo tome un valor mínimo.

b) En el programa hemos observado que obtenemos máximo pero no mínimo. Ante este resultado, si observamos los conjuntos de soluciones factibles del programa nos encontramos que el conjunto $A$ es no acotado. Precisamente la no acotación de $A$ es la que ha ocasionado que no exista mínimo.

\section{CONCLUSIONES}

1. El teorema de Weierstrass nos da la existencia de máximos y mínimos globales en el estudio de la resolución de programas matemáticos.

2. El teorema de programación convexa nos da la convexidad del programa en el que todo óptimo local sea global.

\section{BIBLIOGRAFÍA}

Apóstol, T.: Calculus, Vols. I y II, $2^{\mathrm{a}}$ edición, Reverté, 1973.

Balbas, A. y Gil, J.A.: Programación matemática, Editorial AC, 1987.

Chiang, A.: Métodos fundamentales de economia matemática, $3^{\text {a }}$ edición, McGraw Hill, 1987.

Guerrero Casas, F.: Curso de Optimización: Programación matemática, ariel economía, 1997. Intriligator, M.: Optimización matemática y teoria económica, Prentice-Hall, 1973. 